\title{
Correspondence on Lovell et al.: identification of chicken genes previously assumed to be evolutionarily lost
}

\author{
Susanne Bornelöv ${ }^{1,2}$, Eyal Seroussi ${ }^{3}$, Sara Yosefi ${ }^{3}$, Ken Pendavis ${ }^{4}$, Shane C. Burgess ${ }^{4}$, Manfred Grabherr ${ }^{1,5}$, \\ Miriam Friedman-Einat ${ }^{3^{*}}$ (i) and Leif Andersson ${ }^{1,6,7^{*}}$
}

Please see related Research article: http://dx.doi.org/10.1186/s13059-014-0565-1 and Please see response from Lovell et al: https:/www.dx.doi.org/

$10.1186 /$ s13059-017-1234-y

\begin{abstract}
Through RNA-Seq analyses, we identified 137 genes that are missing in chicken, including the long-sought-after nephrin and tumor necrosis factor genes. These genes tended to cluster in GC-rich regions that have poor coverage in genome sequence databases. Hence, the occurrence of syntenic groups of vertebrate genes that have not been observed in Aves does not prove the evolutionary loss of such genes.
\end{abstract}

A recent paper reported that 274 protein-encoding genes were missing from sequencing data from 60 bird species [1]. Most of them were organized in conserved syntenic clusters in non-avian vertebrates, suggesting that their loss in the avian lineage had occurred through genomic deletions of gene blocks. This hypothesis was supported by another study reporting that 640 protein-encoding genes were missing from 48 bird genomes [2]; the authors of this second study made a similar suggestion that large segmentally deleted regions had been lost during microchromosome evolution in birds. However, our recent discovery of leptin genes with $\sim 70 \%$ GC content in chicken and duck [3], and the new identification of 89 GC-rich genes [4], suggested an alternative hypothesis of a technical barrier to explain the 'missing genes'. To further explore this, RNA-Seq data from visceral fat, hypothalamus, and pituitary tissues from two types of chickens,

\footnotetext{
* Correspondence: miri.einat@mail.huji.ac.il; leif.andersson@imbim.uu.se ${ }^{3}$ Agricultural Research Organization, Volcani Center, Rishon LeZion, Israel ${ }^{1}$ Science for Life Laboratory, Department of Medical Biochemistry and Microbiology, Uppsala University, Uppsala SE-751 23, Sweden Full list of author information is available at the end of the article
}

broilers and layers (Additional file 1: Table S1), were used for de novo transcriptome assembly and identification of novel genes.

The initial set of 588,683 transcripts obtained using Trinity [5] was reduced to 257,700 after removing transcripts that were expressed at low levels. We mapped the transcripts to the chicken reference genome build consistent with the previous studies [1,2] using Blat and Blast, and retained 8395 sequences without alignments. These transcripts were then characterized on the basis of sequence similarity to known genes in other vertebrates using the Trinotate pipeline (https://trinotate.github.io), which searches for sequences encoding known protein domains, transmembrane domains, and signal peptides (Additional file 1: Tables S2 and S3a). Genes that were already known in chicken were removed by comparing their gene symbols with those in Ensembl (release 80), RefSeq, and Entrez Gene, resulting in 1878 novel gene-candidate transcripts representing 1063 genes (Additional file 1: Tables S3b and S4).

To increase specificity and to remove multiple transcript isoforms, we tested each transcript by reciprocal Blastn against the full transcriptome assembly (588,683 transcripts), and Blastx against the set of coding sequences predicted by TransDecoder (https://transdecoder.github.io), consisting of 111,457 sequences. The remaining set yielded 194 transcripts encompassing 190 distinct high-confidence genes (Additional file 1: Table S5). Through Blastn, we found that 55 loci had already been recovered as annotated genes in an updated genome build (Galgal5) released after the previous studies. In addition, 47 genes mapped to the genome but lacked annotations, while another 51 genes were annotated as uncharacterized or putative proteins (Additional file 1: Table S6). One 
discrepancy in annotation between our genes and Galgal5 was observed for the RSAD1 transcript, which was annotated as MYCBPAP in Galgal5. Closer inspection revealed that these two genes, which are close neighbors in the human genome, have been mistakenly merged into MYCBPAP in Galgal5. Therefore, we considered RSAD1 as a novel annotation (Additional file 1: Table S6).

Among the remaining 38 genes (Additional file 1: Table S6) with no sequence similarity to any genome build are the tumor necrosis factor (TNF) and nephrin (NPHS1), which have been reported as missing from birds in several studies (Table 1) but which are critically important in vertebrate biology and have extensively been studied in non-avian vertebrates (there are more than 130,000 publications in PubMed on TNF and 1300 on NPHS1). These genes were subjected to fullcDNA-sequence determination, exon characterization, RT-PCR validation, and expression profiling using RNA-Seq data from red junglefowl (Additional file 2: Figures S1 and S2; Additional file 2: Tables S9 to S12). The similarity in sequences, exon-intron junctions, and characteristic expression profiles confirmed the identification of chicken NPHS1 and TNF, thus resolving the long discussion as to why these genes have been missing from the genome assembly despite their established essential biological function in other species (for examples, see [6-12]).
Mass spectrometry analysis of fat tissue from the same chickens confirmed the identification of $M E P C E$, NPC1L1, PHF1, MRPS18, and SF3B2 at $P<0.01$, and the expression of AMIGO1, CYAB, FKBP11, MGAT1, MOGS, MRI1, MTX1, POLR3D, PEA15, and TXNIP at $P<0.05$ (Additional file 1: Tables S4, S5, and S8). To further validate the novel genes in the context of species phylogeny, we selected 11 genes with complete coding sequences predicted by TransDecoder (Additional file 3: Table S13) and at least four reported orthologous protein sequences in the NCBI protein database, for analysis of protein identity with the predicted chicken amino acid sequence using pBlast. As expected, the relative degrees of sequence identity were inversely correlated with evolutionary distance for most transcripts $(r=-1$ to -0.7$)$, with three exceptions resulting from high conservation.

Comparing these genes to the genes previously reported as missing $[1,2,6]$ recovered 74 overlapping gene symbols (Table 1). A higher proportion of the genes reported missing only in chickens was identified compared to those reported missing in all avian species (15\% and $3-4.5 \%$, respectively). The recovered transcripts had very high GC content (68\%; Additional file 3: Figure S3b), further supporting the hypothesis that many of the genes that are currently missing from the draft genome eluded previous identification because of their high GC content $[3,4]$.

Table 1 Characterization of the novel genes reported missing in previous studies

\begin{tabular}{|c|c|c|c|c|}
\hline Previously reported list & $\begin{array}{l}\text { No. of missing } \\
\text { genes }\end{array}$ & $\begin{array}{l}\text { Found in our } \\
\text { intermediate set }\end{array}$ & $\begin{array}{l}\text { Found in our } \\
\text { high-confidence list }\end{array}$ & Gene symbols \\
\hline $\begin{array}{l}\text { Predicted absent in } \\
\text { birds [1] }\end{array}$ & 274 & $36(13 \%)$ & $8(3 \%)$ & $\underline{\text { FLT3LG }}, \underline{L P P R 2}, \underline{\text { NPHS1}}, \underline{P L C B 3^{\mathrm{a}}}, \underline{\text { PRSS8}}, \underline{\mathrm{RCN} 3}, \underline{\text { TRMT1}}, \underline{\text { TSPAN31 }}$ \\
\hline $\begin{array}{l}\text { Predicted missing in } \\
\text { chickens but not in } \\
\text { all birds [1] }\end{array}$ & 336 & $152(45 \%)$ & $50(15 \%)$ & 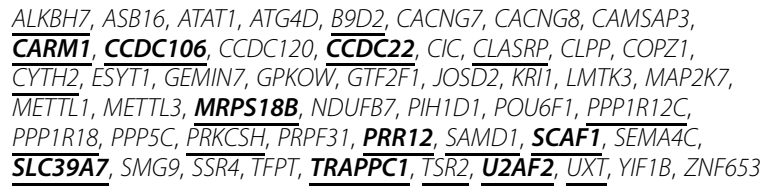 \\
\hline $\begin{array}{l}\text { Predicted absent in } \\
\text { birds [2] }\end{array}$ & 640 & $100(16 \%)$ & $29(4.5 \%)$ & 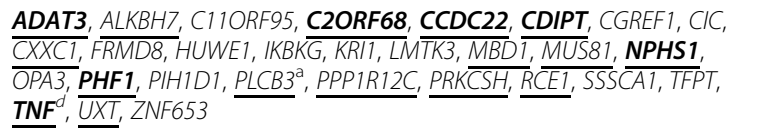 \\
\hline $\begin{array}{l}\text { Predicted absent by } \\
\text { both studies }[1,2]\end{array}$ & 99 & $7(7 \%)$ & $2(2 \%)$ & $\underline{N P H S 1^{c}},{\underline{P L C B 3^{a}}}^{a}$ \\
\hline Lost adipokines [6] & 4 & $1(25 \%)$ & $1(25 \%)$ & $T N F^{\mathrm{d}}$ \\
\hline
\end{tabular}

Eleven genes are shared between row 2 (Lovell et al. [1]) and row 3 (Zhang et al. [2]): ALKBH7, CCDC22, CIC, KRI1, LMTK3, PIHID1, PPP1R12C, PRKCSH, TFPT, UXT, and ZNF653

a $P L C B 3$ was selected manually from the intermediate list of novel genes as a dropout due to misannotation of its quail (Coturnix japonica) ortholog (LOC107307599), demonstrating that the intermediate gene list (Additional file 1: Table S4) may contain additional novel genes

based on the genes listed in Tables S4a, S4b, S6a, and S6b in Lovell et al. [1]

'Also reported missing in other publications (e.g. [7, 14])

${ }^{\mathrm{d}}$ Also reported missing also in Zhang et al. [2] and in additional publications (e.g. [10, 15])

(i) Bold and underlined, (ii) underlined, (iii) underlined by dashed line, and (iv) non-underlined symbols represent (i) novel sequences with no sequence similarity in any genome build, (ii) sequences present in Galgal5 but lacking annotation, (iii) sequences present in Galgal5 as uncharacterized or putative, or (iv) sequences present and annotated in Galgal5, respectively 
When exploring the location of novel genes recovered by the updated genome build, we observed that most genes (76\%) were located on unplaced scaffolds, probably representing uncharacterized microchromosomes. Among those that mapped to known chromosomes, the majority (80\%) were localized to microchromosomes, which are estimated to contain $50 \%$ of protein-coding genes in chickens [13]. Surprisingly, many of the mapped genes appeared in clusters. Mapping positions of the human orthologs demonstrated that the organization of $80 \%$ of the mapped novel genes was in syntenic clusters (Table 2). The strong tendency of these novel genes to cluster indicated their location in recalcitrant chromosomal regions with high GC content, primarily on microchromosomes. The methods used in this study are detailed in Additional file 4: Detailed materials and methods.

\section{Conclusions}

Our RNA-Seq study, combined with extensive bioinformatics analysis, recovered 191 novel genes that were missing from previous chicken assemblies, 38 of which are still not present in the most recent genome build (Galgal5), as well as an additional 47 that are at least partially present in Galgal5 but lacking proper annotation. The high GC content (68\% on average), the microchromosomal location of the majority of the novel genes $(80 \%)$ covered by Galgal5, and their high tendency to cluster into syntenic blocks $(80 \%)$ suggest that the novel genes were not found in earlier analyses because of their position in GC-rich gene clusters, rather than due to chromosomal fragmentation and loss. In addition, the identification and characterization of NPHS1 and TNF, which are expected to be essential for avian physiology, and which are still missing from the latest genome build,

Table 2 Overview of novel genes missing from the Galgal4 assembly but present in Galgal5

\begin{tabular}{|c|c|c|c|c|c|c|}
\hline \multirow[t]{2}{*}{ Trinity ID } & \multirow{2}{*}{$\begin{array}{l}\text { Predicted } \\
\text { gene }\end{array}$} & \multicolumn{3}{|c|}{ Galgal5 mapping } & \multirow[t]{2}{*}{ Human ortholog (hg38) } & \multirow[t]{2}{*}{ Cluster $^{a}$} \\
\hline & & Genes & Chromosome & Coordinates & & \\
\hline c192514_g2_i1 & RRS1 & RRS1 & chr2 & $115,487,692-115,488,635$ & chr8:66,429,028-66,430,733 & - \\
\hline c144374_g1_i1 & KHK & KHK & chr3 & $104,952,675-104,954,000$ & chr2:27,086,747-27,100,751 & 1 \\
\hline C150768_g1_i3 & CGREF1 & CGREF1 & chr3 & $104,955,106-104,955,990$ & chr2:27,100,594-27,119,103 & 1 \\
\hline c191309_g1_i2 & ANKRD66 & LOC101750448 & chr3 & $110,320,024-110,320,850$ & chr6:46,746,917-46,759,506 & - \\
\hline c190219_g1_i1 & $A D O$ & $A D O$ & chr6 & $8,089,943-8,090,591$ & chr10:62,804,857-62,808,483 & - \\
\hline c165457_g1_i6 & $A B H D 14 B$ & LOC107056876 & chr12random_Scaffold5645 & $10,835-12,580$ & chr3:51,968,510-51,983,409 & - \\
\hline c181867_g2_i3 & RSAD1 & MYCBPAP & chr18 & $10,429,164-10,430,334$ & chr17:50,508,384-50,531,497 & - \\
\hline c160691_g1_i2 & $B O L A 3$ & $B O L A 3$ & chr22 & $2,880,009-2,880,858$ & chr2:74,135,398-74,147,994 & 2 \\
\hline c178063_g1_i8 & SEMA4C & SEMA4C & chr22random_Scaffold1011 & $444-4,447$ & chr2:96,859,716-96,869,971 & 2 \\
\hline c156624_g2_i1 & CIART & CIART & chr25 & $2,384,775-2,385,633$ & chr1:150,282,543-150,287,093 & 3 \\
\hline c165802_g2_i1 & CRTC2 & CRTC2 & chr25 & $2,075,046-2,076,072$ & chr1:153,947,675-153,958,625 & 3 \\
\hline c189493_g2_i1 & C17orf96 & LOC107055293 & chr27 & $4,355,476-4,355,902$ & chr17:38,671,703-38,675,421 & 4 \\
\hline c151660_g2_i1 & $K R / 1$ & LOC107055293 & chr27 & $4,357,140-4,357,428$ & chr19:10,553,078-10,566,037 & 4 \\
\hline c167546_g1_i3 & FBXW9 & FBXW9 & chr30random_Scaffold7361 & $448-2,027$ & chr19:12,688,917-12,696,643 & 5 \\
\hline c160528_g1_i2 & DHPS & DHPS,WDR83 & chr30random_Scaffold7361 & $2,298-5,407$ & chr19:12,675,721-12,681,902 & 5 \\
\hline c150426_g1_i4 & YIF1B & YIF1B & chr32random_Scaffold22667 & $160-217$ & chr19:38,305,118-38,315,963 & 6 \\
\hline c167964_g1_i2 & B9D2 & - & chr32random_Scaffold15198 & $71-292$ & chr19:41,354,421-41,364,173 & 6 \\
\hline c164748_g1_i1 & OPA3 & OPA3 & chr32random_Scaffold826 & $46,400-48,070$ & chr19:45,546,281-45,584,819 & 6 \\
\hline c148689_g1_i2 & SNRPD2 & SNRPD2 & chr32random_Scaffold19601 & $235-1,401$ & chr19:45,687,454-45,692,333 & 6 \\
\hline c163802_g1_i1 & GRASP & GRASP & chr33 & $1,916-6,474$ & chr12:52,006,940-52,015,864 & 7 \\
\hline c178972_g2_i2 & ESYT1 & ESYT1 & chr33 & $679,134-685,279$ & chr12:56,128,056-56,144,671 & 7 \\
\hline c171696_g1_i1 & $A P O F$ & APOF & chr33 & $776,046-776,629$ & chr12:56,360,569-56,362,823 & 7 \\
\hline c100851_g1_i1 & HOXC4 & HOXC4 & chr33 & $1,095,140-1,096,547$ & chr12:54,016,931-54,055,327 & 7 \\
\hline c186414_g2_i1 & COPZ1 & COPZ1 & chr33 & $1,170,192-1,174,833$ & chr12:54,325,127-54,351,849 & 7 \\
\hline c146677_g1_i1 & DAZAP2 & - & chr33 & $1,573,156-1,573,299$ & chr12:51,238,292-51,243,933 & 7 \\
\hline
\end{tabular}

${ }^{\mathrm{a}}$ This column indicates clusters of neighboring genes that are largely supported by the human orthologs 
emphasizes the importance of striving towards a repertoire of known and characterized genes that is as complete as possible.

\section{Additional files}

Additional file 1: Overview of the RNA-Seq data and filtration of the novel gene candidates. Table S1. Information about the RNA-Seq data. Table S2. The initial set of 2810 candidate novel transcripts. Table S3. Annotation, characterization, and filtering of the novel transcripts. Table $\mathbf{S 4}$ The intermediate set of 1878 transcripts representing 1063 candidate novel genes. Table S5. The high-confidence set of 194 transcripts representing 191 novel genes. Table S6. The 191 novel genes not included in Galgal4; 54 of these are correctly annotated while 137 are missing or lack correct annotation in Galgal5. Table S7. Characterization of the novel genes according to predicted cellular localization. Table S8. Identification of the novel genes in Galgal5 genome assembly and by Mass-Spec analysis in adipose tissue. (XLS $2362 \mathrm{~kb}$ )

Additional file 2: Characterization of NPHS1 and TNF. Figure S1. Predicted full length cDNA sequence of NPHS1 and its characterization. Figure S2. Predicted full length CDNA sequence of TNF and its characterization. Table S9. Coding sequence of chicken NPHS1 and TNF predicted transcripts. Table S10. List of NPHS1 and TNF exons in human, turtle, and chicken. Table S11. List of primers used for RT-PCR. Table S12. Probes used for expression profiling in the Sequence Read Archive (SRA) database. (PDF 1672 kb)

Additional file 3: Characterization of the high confidence novel genes. Table S13. Phylogenetic analysis of representative novel genes. Figure S3. Characterization of the novel transcripts. (PDF $232 \mathrm{~kb}$ )

Additional file 4: Detailed materials and methods. Animals and tissue sampling. RNA-seq. Bioinformatic analysis. RT-PCR. Mass spectrometry analysis (MS). (PDF $283 \mathrm{~kb}$ )

\section{Abbreviations}

NPHS1: Nephrin; TNF: Tumor necrosis factor

\section{Acknowledgment}

We thank Mr Mark Ruzal for growing the chickens. The study was supported by the ERC project BATESON (awarded to LA), Israel Academy of Sciences 876/14, and by the Chief Scientist of the Israeli Ministry of Agriculture 0469/14 (awarded to MFE and ES).

\section{Availability of data and materials}

The raw sequences that were used to build the trinity transcripts of the novel genes, as well as the CDNA sequences of the chicken NPHS1 and chicken and turkey $T N F$, are available in the ENA BioProject repository [PRJEB13623, www.ebi.ac.uk/ena/data/view/PRJEB13623].

\section{Authors' contributions}

SB performed the transcript assembly and produced the novel gene lists. ES extended and characterized the NPHSI and TNF predicted CDNAs and proteins. MFE and SY performed the biological experiments, prepared the RNA for sequencing, confirmed the deduced CDNA sequences of NPHS 1 and TNF by RT-PCR, and performed the NPHS1 and TNF expression profiling. KP and SCB performed the MS analysis. MG helped to design the bioinformatic approaches. MFE, SB, and LA designed the experiments and wrote the manuscript. All authors read and approved the final manuscript.

\section{Competing interests}

The authors declare that they have no competing interests.

\section{Ethics approval and consent to participate}

All animal procedures were carried out in accordance with the National Institutes of Health Guidelines on the Care and Use of Animals and Protocol IL536/14, which was approved by the Animal Experimentation Ethics Committee of the Agricultural Research Organization, Volcani Center, Rishon, Israel.

\section{Publisher's Note}

Springer Nature remains neutral with regard to jurisdictional claims in published maps and institutional affiliations.

\section{Author details}

${ }^{1}$ Science for Life Laboratory, Department of Medical Biochemistry and Microbiology, Uppsala University, Uppsala SE-751 23, Sweden. ${ }^{2}$ Present Address: Wellcome Trust Medical Research Council Stem Cell Institute, University of Cambridge, Cambridge CB2 1QR, UK. ${ }^{3}$ Agricultural Research Organization, Volcani Center, Rishon LeZion, Israel. ${ }^{4}$ College of Agriculture and Life Sciences, University of Arizona, Tucson, AZ 85721-0036, USA ${ }^{5}$ Bioinformatics Infrastructure for Life Sciences, Uppsala University, Uppsala, Sweden. ${ }^{6}$ Department of Animal Breeding and Genetics, Swedish University of Agricultural Sciences, Uppsala SE-750 07, Sweden. ${ }^{7}$ Department of Veterinary Integrative Biosciences, College of Veterinary Medicine and Biomedical Sciences, Texas A\&M University, College Station, TX 77843-4458, USA.

Published online: 14 June 2017

\section{References}

1. Lovell PV, Wirthlin M, Wilhelm L, Minx P, Lazar NH, Carbone L, et al. Conserved syntenic clusters of protein coding genes are missing in birds. Genome Biol. 2014:15:565.

2. Zhang G, Li C, Li Q, Li B, Larkin DM, Lee C, et al. Comparative genomics reveals insights into avian genome evolution and adaptation. Science. 2014;346:1311-20.

3. Seroussi E, Cinnamon Y, Yosefi S, Genin O, Smith JG, Rafati N, et al. Identification of the long-sought leptin in chicken and duck: expression pattern of the highly GC-rich avian leptin Fits an autocrine/paracrine rather than endocrine function. Endocrinology. 2016;157:737-51.

4. Hron T, Pajer P, Paces J, Bartunek P, Elleder D. Hidden genes in birds. Genome Biol. 2015:16:164

5. Grabherr MG, Haas BJ, Yassour M, Levin JZ, Thompson DA, Amit I, et al. Full-length transcriptome assembly from RNA-Seq data without a reference genome. Nat Biotechnol. 2011;29:644-52.

6. Dakovic $N$, Terezol M, Pitel F, Maillard V, Elis S, Leroux S, et al. The loss of adipokine genes in the chicken genome and implications for insulin metabolism. Mol Biol Evol. 2014;31:2637-46.

7. Miner JH. Life without nephrin: it's for the birds. J Am Soc Nephrol. 2012:23:369-71

8. Wajant $H$, Pfizenmaier $K$, Scheurich P. Tumor necrosis factor signaling. Cell Death Differ. 2003;10:45-65.

9. Wagner N, Morrison H, Pagnotta S, Michiels JF, Schwab Y, Tryggvason K, et al. The podocyte protein nephrin is required for cardiac vessel formation. Hum Mol Genet. 2011:20:2182-94.

10. Uysal B, Donmez O, Uysal F, Akaci O, Vuruskan BA, Berdeli A. Congenital nephrotic syndrome of NPHS1 associated with cardiac malformation. Pediatr Int. 2015;57:177-9.

11. Li X, Chuang PY, D'Agati VD, Dai Y, Yacoub R, Fu J, et al. Nephrin preserves podocyte viability and glomerular structure and function in adult kidneys. J Am Soc Nephrol. 2015;26:2361-77.

12. Kestila M, Lenkkeri U, Mannikko M, Lamerdin J, McCready P, Putaala H, et al. Positionally cloned gene for a novel glomerular protein - nephrin - is mutated in congenital nephrotic syndrome. Mol Cell. 1998;1:575-82.

13. Smith J, Bruley CK, Paton IR, Dunn I, Jones CT, Windsor D, et al. Differences in gene density on chicken macrochromosomes and microchromosomes. Anim Genet. 2000;31:96-103.

14. Yaoita E, Nishimura $H$, Nameta M, Yoshida Y, Takimoto H, Fujinaka $H$, et al. Avian podocytes, which lack nephrin, use adherens junction proteins at intercellular junctions. J Histochem Cytochem. 2016:64:67-76.

15. Magor KE, Miranzo Navarro D, Barber MR, Petkau K, Fleming-Canepa X, Blyth GA, Blaine AH. Defense genes missing from the flight division. Dev Comp Immunol. 2013:41:377-88. 